The top quark reconstructed mass distribution for the $b$-tagged $W+\geq 4$-jet events (solid line) Also shown are the background shape (dotted line) and the sum of the background plus ti Monte Carlo calculations (dashed line) for a top quark mass of $175 \mathrm{GeV} / \mathrm{c}^{2}$, with the background constrained to the calculated value of $6.9_{-1.9}^{+2.5}$ events.

mimic, instead, the complex features of biological evolution based on simple rules so that interesting generalisations can be developed. The main finding is that most models demonstrate self-organized critical behaviour, as in sand piles, earthquakes, etc. Indeed, numerous models lead to an unstable critical state. When perturbing this quasi-steady state, the evolution process always self-organizes into the same critical steady state having, as a consequence, the same appearance. The dynamics of the perturbations show power-law behaviour. Selforganized criticality of this type is sometimes said to be on "the edge of chaos" since a small perturbation can cause an accidental and unpredictable endless dissipation.

Postulating the self-organization between chaos and order as the basic rule for life evolution is plausible because this behaviour can only evolve if small mutations have small but finite effects. If they have no effect (i.e., they are deep in the ordered region), mutations cannot drive evolution; for catastrophic effects (i.e., deep in the chaotic region), a single mutation can destroy almost everything. This leads one to speculate whether the physical and universal rules for quarks and atoms which lead to self-organization into a critical state also apply to the emergence of complexity and living organisms.

The physics community's contribution to understanding evolution will bear much upon the study of self-organized criticality presented by "artificial" (but biologically motivated) computer systems. The concept of critical self-organization is recent and a general formalism of such behaviour is still lacking. Ideas will take time to mature, hopefully not on a geological timescale.

One should, for example, look whether additional biological constraints lead to the emergence of evolution process(es) from self-organized criticality. Fundamental and philosophical questions about evolution and the origins of life, as well as how many types of life can exist, are raised as a result. Computers can search for some answers. It is also necessary to identify which "perturbations" force natural systems to evolve towards a particular "steady-state". Finally, it will be important, but very difficult, to study transient effects that today defy a systematic description

[1] Vandewalle N. \& Ausloos M., "Evolution motivated computer models", in Ann. Rev. Comp. Phys., Ed.: D. Stauffer (World Scientific, 1996) Vol. 3; in press.

[2] Vandewalle N. \& Ausloos M., J. Phys. I (1995), in press.

\title{
Top Quark Confirmed
}

On 24 February 1995, both the CDF and D0 Collaborations at the Fermilab Tevatron Collider submitted papers [1] detailing new and convincing evidence for the existence of the top quark. These papers confirm with significantly increased statistics evidence from CDF announced in April 1994 [2].

Top quarks are produced as particle-antiparticle pairs in $\overline{\mathrm{p}}-\mathrm{p}$ collisions, and because of their large mass ( $>170$ times that of the proton) are produced very rarely. From $\approx 10^{8}$ events recorded on tape by the experiments, the CDF and DO Collaborations reported respectively 43 and 17 events consistent with t-quark production. The new results correspond to a data sample corresponding to an integrated luminosity of $\approx 117 \mathrm{pb}^{-1}$, as compared with the $\approx 19 \mathrm{pb}^{-1}$ used for the previous CDF analysis. This huge increase in statistics has been made possible by continued and higher intensity operation of the Fermilab collider.

Top quarks decay to a W-boson and a b-quark, and the W-boson decays into either leptons or quarks. Events for which both W-bosons decay into leptons (e or $\mu$ ) result in a striking high transverse momentum $P_{\mathrm{T}}$ opposite-sign dilepton $(\mathrm{e}, \mu)$ signature with associated missing transverse energy from undetected neutrinos and at least $2 \mathrm{~b}$-quark jets. Both experiments are able to detect this production and decay chain with good efficiency. The CDF experiment reports a

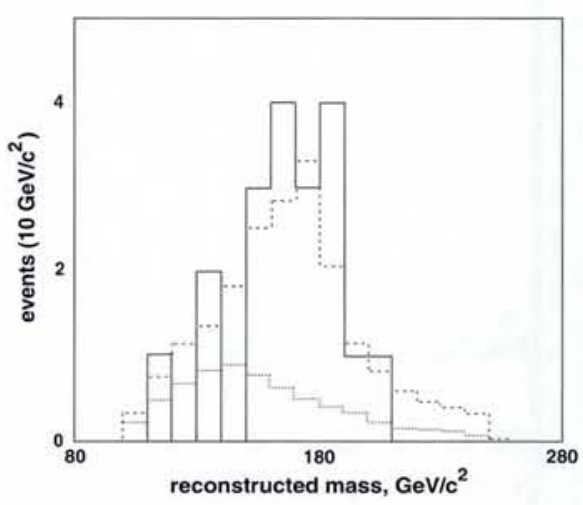

valent result in D0 is 17 lepton $+\geq 3$ jet events with a background estimate of 3.9 \pm 0.6 events (this sample includes 6 low- $P_{T}$ muon tags from semileptonic b-decay, with a background of $1.2 \pm 0.2$ events).

The event samples and associated background uncertainties noted above reflect the differing capabilities of the two experiments; the CDF experiment uses to advantage its excellent secondary vertex reconstruction capability (SVX), while the DO experiment benefits from its outstanding muon-detector and calorimeter coverage.

From these data the probability of a fluctuation of the background to produce the observed signal is estimated as $<2 \times 10^{-6}$, corresponding to 4.6 (4.8) standard deviations for the DO and CDF experiments, respectively. Also in agreement, the $t-\tilde{t}$ production cross-section in $\bar{p}-p$ interactions at the centre-of-mass energy $\sqrt{ } s=1.8 \mathrm{TeV}$ is measured to be respectively $6.4 \pm 2.2 \mathrm{pb}$ and $6.8_{-2.4}^{+3.6} \mathrm{pb}$, for the quoted $\mathrm{t}$-quark mass values. The results are in reasonable agreement with theoretical expectations.

Several concerns of the previous CDF analysis, e.g., the measured cross-section and the excess of $W+4$-jet events compared with $\mathrm{W}+$ jet $\mathrm{QCD}$ predictions, have been resolved by the increased statistics.

From the sample of $W+4$-jet events, each experiment has performed a t-quark mass reconstruction. The CDF experiment estimates the t-quark mass to be $176 \pm$ 8 (stat) \pm 10 (sys) $\mathrm{GeV} / \mathrm{c}^{2}$, to be compared with an estimated mass given as $174 \pm 10$ (stat) \pm 13 (sys) $\mathrm{GeV} / \mathrm{c}^{2}$ in the 1994 data. The t-quark reconstructed mass is shown in the figure. The significant systematic uncertainty results primarily from uncertainties on the mass-dependence of the background, and on uncertainties on the effect of gluon radiation. The challenge within CDF is now to reduce both the statistical and systematic uncertainties on the mass measurement to a level that constrains the allowed values of the Higgs mass (assuming validity of the Standard Model). To this end, each experiment is likely to double its data sample in the current collider run late in 1995. The DO experiments estimates the mass to be $199_{-20}^{+19}$ (stat) \pm 22 (sys) $\mathrm{GeV} / \mathrm{c}^{2}$; this result is not inconsistent with the CDF result.

[1] Abe F. et al., Phys. Rev. Lett. 74 (1995) 2626; Abachi S. et al., Phys. Rev. Lett. 74 (1995) 2632. [2] Abe F. et al., Phys. Rev. D 50 (1994) 2966; Abe F. et al., Phys. Rev. Lett. 73 (1994) 22. See also: Abachi S. et al., Phys. Rev. Lett. 72 (1994) 2138; Bellettini G. \& Clark A.G., Europhys. News 25 (1994) 86.

A.G. Clark, Geneva University 